\title{
Clinical significance of interleukin-10 concentration in the cerebrospinal fluid of patients with primary central nervous system lymphoma
}

\author{
MINGYING GENG $^{1 *}$, YANG SONG $^{1 *}$, HE XIAO $^{1}$, ZHIYU WU $^{2}$, \\ XIAOJUAN DENG ${ }^{3}, \mathrm{CHUAN}^{\mathrm{CHEN}}{ }^{1}$ and $\mathrm{GE} \mathrm{WANG}^{1}$ \\ Departments of ${ }^{1}$ Cancer Center, ${ }^{2}$ Clinical Laboratory and ${ }^{3}$ Radiology, Daping Hospital, \\ Army Medical University, Chongqing 400042, P.R. China
}

Received March 31, 2020; Accepted September 11, 2020

DOI: $10.3892 / \mathrm{ol} .2020 .12263$

\begin{abstract}
The diagnostic and prognostic evaluation of primary central nervous system lymphoma (PCNSL) is challenging due to the lack of sensitive biomarkers. The present study aimed to evaluate the value of interleukin (IL)-10 in this context. Between October 2016 and December 2018, 91 patients with suspected intracranial neoplasms were recruited, and the concentrations of IL-10 or IL- 6 in both the cerebrospinal fluid (CSF) and blood were measured and analyzed by the Kruskal-Wallis test. The correlation between CSF IL-6 or IL-10 levels and tumor size was determined by Spearman's coefficient analysis. The receiver operating characteristic curve was used to evaluate the diagnostic value of CSF IL-6 and IL-10 levels. Median progression-free survival (PFS) and overall survival time were calculated using Kaplan-Meier survival analysis. Among the 91 patients, 3 were diagnosed with PCNSL on the basis of neuroimaging data and CSF IL-10 levels. A total of 35 cases were verified to show diffuse large B-cell lymphoma on histological assessment, 17 of which were diagnosed as PCNSL by MRI. The median PFS and OS were 8.00 months [95\% confidence interval (CI), 3.94-12.06) and 17.5 months $(95 \% \mathrm{CI}, 11.55-23.45)$ respectively in the 12 PNCSL cases with regular follow up. The diagnostic efficiency of serum IL-6 levels was lower than that of serum IL-10 levels $(\mathrm{P}=0.030)$, which, in turn, was lower than that of CSF IL-10 levels $(\mathrm{P}<0.001)$. The decline and increase in CSF IL-10
\end{abstract}

Correspondence to: Dr Chuan Chen or Dr Ge Wang, Department of Cancer Center, Daping Hospital, Army Medical University, 10 Changjiang Branch Road, Yuzhong, Chongqing 400042, P.R. China

E-mail: sinkriver@126.com

E-mail: coco7520@163.com

*Contributed equally

Key words: primary central nervous system lymphoma, cerebrospinal fluid, serum, interleukin 6, interleukin 10 levels was concurrent with improvement and deterioration in manifestation, respectively, which predated the MRI variation. High CSF IL-10 levels indicated low Karnofsky performance scale scores and shortened PFS times. CSF IL-10 levels higher than $1,000 \mathrm{pg} / \mathrm{ml}$ signified disease progression. CSF IL-10 levels could be a sensitive biomarker guiding the differential diagnosis, early recurrence detection, prognostic evaluation and therapeutic strategy establishment in cases of PCNSL.

\section{Introduction}

Primary central nervous system lymphoma (PCNSL) belongs to the group of non-Hodgkin's lymphomas (NHLs) occurring outside the lymph nodes, of which $90 \%$ are forms of diffuse large B-cell lymphoma (DLBCL) (1). These tumors account for $3-5 \%$ of all cases of primary brain tumors globally and are characterized by single or multiple intracranial lesions without extracranial dissemination (1). Over the past 25 years, the incidence of PCNSL has quadrupled, especially in the human immunodeficiency virus (HIV)-infected population (1).

Due to the lack of specific neuroimaging features, the differential discriminative diagnosis of PCNSL as a condition distinct from other brain tumors, such as glioma and metastatic encephaloma, is quite difficult, and misdiagnoses are likely to occur $(2,3)$. An analysis of 46 PCNSL cases with a pathological primary diagnosis in Daping Hospital (Chongqing, China) between 2007 and 2016 showed that the agreement between MRI data and pathological evidence was only $39.1 \%$ (unpublished data). The sensitivity (88\%) and specificity $(86 \%)$ of positron emission tomography-computed tomography (PET-CT) are reported to be higher than those of MRI (4). However, PET-CT examination is usually delayed due to the rapid development of neurological symptoms stemming from multifocal and diffuse invasive growth of PCNSL. Additionally, the role of surgery in PCNSL is usually limited to stereogenic biopsy to allow a clear pathological diagnosis (5). In addition, surgical resection increases the risk of permanent neurological impairment and prolongs the time from diagnosis to chemotherapy administration (6). HD-MTX is currently considered the basis for the application of systemic chemotherapy in newly diagnosed PCNSL (7). This treatment 
regimen is significantly different from that of other solid tumors, such as glioma for which the temozolomide (TMZ) based concurrent radiotherapy has been well established (8).

Therefore, on the basis of MRI and PET-CT examinations, there is an urgent need to identify novel high-sensitivity, high-specificity biomarkers that can be used to quickly and simply evaluate patient diagnoses and differential diagnoses, screen treatment regimens, evaluate efficacy and prognosis, and monitor for early recurrence.

Neopterin (Npt), interleukin (IL)-6 or IL-10 and microRNA are considered to play roles in the diagnosis of PCNSL, with relatively high sensitivity and specificity (9-12). However, in our retrospective study on CSF Npt, this biomarker showed a false-positive ratio of $16 \%$, and the accuracy of clinical diagnosis was greatly reduced (13). IL-6 is a potent pluripotent inflammatory factor produced by cells that promotes the growth and differentiation of lymphocytes (14); it is a powerful growth and differentiation factor for lymphocytes that is secreted by various cells, such as benign and malignant B lymphocyte, monocytes, macrophages, fibroblasts and hepatocytes $(15,16)$. In a previous study, a primary B-cell lymphoma cell line was found to produce IL-6 in an autocrine manner, promoting maturation of the secreting B cells into plasma cells that produce antibodies. In NHL, IL-10 plays a central role in the growth of B lymphocytes and chemotactic factors, and can induce cells to release immunoglobulin (17), which has been proven to be associated with the occurrence and prognosis of lymphoid malignancies $(18,19)$. IL-10 is secreted by type 2 helper cells, monocytes, macrophages and B lymphocytes in normal and malignant lymphoid tissues, which promotes the growth and differentiation of lymphocytes, and activates B cells to release immunoglobulin (20-22). IL-10 is considered to be the strongest immunosuppressive or anti-inflammatory cytokine, and plays a variety of roles in the development and progression of lymphoma $(20,22,23)$. Therefore, the purpose of the present study was to investigate the potential of CSF IL-6 and IL-10 levels as novel biomarkers for PCNSL diagnosis, differential diagnosis, efficacy evaluation, treatment prognosis estimation and early recurrence monitoring.

\section{Materials and methods}

Patient recruitment. The present study was prospective in nature, and patients with suspected PCNSL based on neuroimaging data obtained between October 2016 and December 2018 were recruited as subjects to undergo CSF IL-6 and IL-10 examinations. The sampling procedure and the objective of the study were explained to the patients and their guardians or family members, and written informed consent was obtained. All baseline characteristics including gender, age, Karnofsky performance scale (KPS) (24), results from Pandy's test (25), WHO grade (26), chemotherapy administration and radiotherapy scheme was extracted from clinical records. The study protocol was approved by The Ethics Committee of Daping Hospital.

Patients were included if they met the following criteria: i) Preoperative MRI or CT findings suggesting PCNSL, a malignant brain tumor or cerebral inflammation; and ii) HIV-seronegative status without a history of autoimmune diseases or organ transplantation. The exclusion criteria were as follows: i) Age $<12$ years; ii) history of trauma; iii) persistent high intracranial pressure; iv) CT or ultrasonic findings indicating diseases outside the CNS; v) other contraindications of lumbar puncture; and vi) HIV-seropositive status. Patients were withdrawn from the study if they met the following criteria: i) Pathological examination indicating the absence of PCNSL, with follow-up examinations being halted as a result; ii) withdrawal of consent by the patients or their family members; iii) contraindications of lumbar puncture; and iv) coma or death of the patient. Patients histologically confirmed with intracranial metastatic tumor or glioma comprised the control group. These patients were treated according to NCCN guidelines (27).

CSF collection and IL-6 or IL-10 measurement. Within 2 weeks of the neuroimaging examinations and 1 week before the operation, the patients underwent a lumbar puncture and venipuncture to obtain 2-5 $\mathrm{ml}$ of CSF and $2-5 \mathrm{ml}$ of blood, respectively. After the results of histological examinations confirmed PCNSL (28), the patients underwent the following protocol: The tumor was evaluated by MRI scans before the operation and prior to and during 2-4 courses of chemotherapy (rituximab [R; $375 \mathrm{mg} / \mathrm{m}^{2}$ day (d)1], high-dose methotrexate (H-MTX, $\left.4 \mathrm{~g} / \mathrm{m}^{2}, \mathrm{~d} 1\right)$ and TMZ $\left(150 \mathrm{mg} / \mathrm{m}^{2}, \mathrm{~d} 1-5\right)$. At 1 week prior to and after the MRI examinations, CSF and blood were collected again using the aforementioned techniques, and both IL-6 and IL-10 concentrations were measured. For patients with severe encephaledema, an intravenous infusion of $20 \%$ mannitol was performed for 2-3 days and $30 \mathrm{~min}$ prior to lumbar puncture. The flow rate of CSF was set at $1 \mathrm{drip} / \mathrm{second}$, and the needle was removed after 2-5 $\mathrm{ml}$ of CSF was obtained, after which the patients lay in the supine position without a pillow for 2-4 h. The obtained CSF was equally divided into two parts: One part was used for routine biochemical and cell count analysis, and the other was centrifuged for $10 \mathrm{~min}$ at 2,000 $\mathrm{x} g$ at room temperature and supernatant was stored in $-80^{\circ} \mathrm{C}$ before determination of the IL- 6 or IL-10 concentration.

CSF and blood IL-6 levels were measured by electrochemiluminescence immunoassay using an Automatic Immune Analyzer (Cobas e601, Roche Diagnostics GmbH) with an IL-6 test kit (cat. no. 36459902, Roche Diagnostics $\mathrm{GmbH}$ ), which detects IL-6 within a dynamic range of $1.50-5,000 \mathrm{pg} / \mathrm{ml}$. IL-10 levels were measured by using an chemiluminescence immunoassay analyzer with a IL-10 test kit (cat. no. 0408, Roche Diagnostics $\mathrm{GmbH}$ ), which detects IL-10 within a dynamic range of $1.00-1,000 \mathrm{pg} / \mathrm{ml}$. All the analytical procedures were performed in accordance with the manufacturer's instructions.

Therapeutic regimen determination. A total of 38 patients with PCNSL underwent the following therapeutic and follow-up protocols. Multiple programs devised by different departments at Daping Hospital ran parallel. In total, 26 patients chose induction chemotherapy as the primary treatment, among which 21 received whole-brain radiotherapy, 2 were monitored without further therapy, 1 received stem cell transplantation and 2 were administered TMZ. Another 9 patients chose radiotherapy and 3 chose Gamma Knife radiosurgery as the primary treatment. On the basis of the National Comprehensive Cancer Network (NCCN) Clinical Practice Guidelines 
recommendations in 2018 (27), whole-brain radiotherapy with a dose of 23.4 Gy was performed for patients exhibiting a complete response $\mathrm{CR}$, an irradiation dose up to $45 \mathrm{~Gy}$ was administered around the tumor mass for patients not achieving a CR and 24-36 Gy whole-brain radiotherapy with 45-Gy irradiation around the tumor mass was performed for patients who were unsuitable for chemotherapy.

Parameters and evaluation criteria in MRI imaging. A Siemens 3.0T superconducting MR scanner (Magnetom verio, Siemens Healthineers) with a 32-channel head coil was employed. The scan sequences and parameters were as follows: T1-weighted imaging (T1WI): Repetition time (TR)/echo time (TE), 440/2.48 msec; T2WI: TR/TE, 4,900/96 msec; FLAIR sequence: TR/TE, 8,000/94 msec; diffusion-WI sequence: $\mathrm{b}$ value set at 0 and $1,000 \mathrm{sec} / \mathrm{mm}^{2}$; perfusion-WI sequence: TR/TE, 1,872/30 msec; susceptibility-WI sequence: TR/TE, 27/20 msec; and MRS sequence: TR/TE, 1,700/135 msec. Enhanced scans with Gd-DTPA were conducted on all the patients. Post-processing was performed using the software package NUMARIS/4 (version syngo MR 2004V) included in the Siemens workstation.

Enhanced MRI and IL-6/IL-10 measurements were performed once at each of the following time points: Before the operation, after the operation, after every 2-4 courses of induction chemotherapy, before radiotherapy and 1 month after radiotherapy. During the follow-up period, these assessments were repeated once every 3 months and a response status assigned. CR indicates that no tumor mass was present on enhanced MRI after therapy. Partial response (PR) indicates that the size of the tumor mass (sum of the maximal diameters of the tumor mass) decreased by at least $50 \%$. Stable disease indicates that the tumor size decreased by the extent below the standard of PR or increased by no more than $25 \%$. Progressive disease indicates that the tumor size increased by more than $25 \%$. Progression-free survival (PFS) is defined as the period from the beginning of therapy to relapse or disease progression, and overall survival (OS) is defined as the period from the beginning of therapy to the last evaluation or death from any cause.

Statistical analysis. The IL-6/IL-10 concentration was measured by laboratory technicians from the Cancer Center laboratory at Daping Hospital who were blinded to the patients' clinical characteristics. The $\chi^{2}$ test was used to compare the basic pathological features between control (non-PCNSL patients) and PCNSL groups. Fisher's exact probability test was adopted when necessary. The concentrations of biochemical indices, as well as the IL-10/IL-6 levels in serum or CSF, were represented by median and interquartile ranges, and the differences between groups were analyzed by using the Kruskal-Wallis test followed by pairwise comparisons with Bonferroni's correction for multiple tests. The correlation between CSF IL-10/IL-6 levels and tumor size was determined by Spearman's rank correlation coefficient. The receiver operating characteristic (ROC) curve and area under curve (AUC) was used to assess the diagnostic efficacy of CSF IL-6 or IL-10 levels for PCNSL. The method of Youden index was used to determine optimal cut-off value (29). Median PFS and OS times, as well as their corresponding $95 \%$ confidence intervals, were determined by Kaplan-Meier survival analysis. $\mathrm{P}<0.05$ was considered to indicate a statistically significant difference. All statistical analyses were performed with SPSS 21.0 software (IBM Corp.).

\section{Results}

Clinical characteristics of the patients. A total of 91 patients with suspected PCNSL on the basis of neuroimaging examinations or clinicians' impressions underwent IL-6/IL-10 measurements and prospective analysis. In 3 patients, the definitive diagnosis was based on neuroimaging and CSF IL-10 results without confirmation through biopsy. Histological examinations confirmed DLBCL in 35 patients and other tumors in the remaining 53 patients, including 22 patients with glioma (13 with grade IV glioblastoma, 5 with anaplastic glioma and 4 with diffuse astrocytoma), 3 with ependymoma, 13 with brain metastasis, 10 with a medulloblastoma/primitive neuroectodermal tumor, 4 with germinoma and 1 with tuberculoma. Among the patients with PCNSL, 20 exhibited multiple lesions on MRI and 12 were followed up regularly. None of the patients showed severe complications such as cerebral hernia.

CSF IL-6 and IL-10 levels in PCNSL were higher than those in other brain tumors. Baseline characteristics of the entire patient population are shown in Table I. There was no significant intergroup difference in sex distribution $(\mathrm{P}=0.097)$ and the number of intracranial lesions $(\mathrm{P}=0.086)$. However, the proportion of female patients, patients with $>3$ lesions or those showing negative results in Pandy's test was higher in the PCNSL group than in the control group. Among the continuous variables, age $(\mathrm{P}=0.001)$, KPS $(\mathrm{P}<0.001)$, serum IL-10 level ( $\mathrm{P}=0.024)$, CSF IL-10 level $(\mathrm{P}<0.001)$, leukocyte count $(\mathrm{P}=0.019), \mathrm{CSF}$ glucose concentration $(\mathrm{P}<0.001)$ and the ratio of IL-10 to IL-6 $(\mathrm{P}<0.001)$ exhibited significant differences between the PCNSL and control groups (Table I). The boxplot in Fig. 1 shows the distribution of serum IL-6, serum IL-10, CSF IL-6 and CSF IL-10 levels in the two groups. In comparison with the control group, the serum and CSF IL-6 levels in the PCNSL group showed no obvious difference; however, both serum and CSF IL-10 levels increased significantly $(\mathrm{P}<0.001$ and $\mathrm{P}=0.024$, respectively).

Contribution of CSF IL-6 and IL-10 to the diagnosis of PCNSL. ROC curve analysis was performed for each parameter that showed significant differences in Table I. As shown in Fig. 2 and Table II, serum IL-10 level ( $\mathrm{P}=0.030), \mathrm{CSF}$ IL-10 level $(\mathrm{P}<0.001), \mathrm{CSF}$ leukocyte count $(\mathrm{P}=0.020), \mathrm{CSF}$ glucose concentration $(\mathrm{P}<0.001)$ and the ratio of IL-10 to IL-6 levels in the CSF $(\mathrm{P}<0.001)$ showed the ability to discriminate patients with PCNSL from those without PCNSL.

Comparison of the diagnostic efficiencies of serum and CSF IL-10 levels showed that the former was substantially inferior to the latter. On the basis of the Youden index, the optimal serum IL-10 cut-off value in all 91 patients was determined to be $2.07 \mathrm{pg} / \mathrm{ml}$, and the sensitivity, specificity, positive predictive value (PPV) and negative predictive value (NPV) of this cut-off value were $59.4,83.3,79.2$, and $65.8 \%$, respectively. By contrast, the cut-off CSF IL-10 value was $10.13 \mathrm{pg} / \mathrm{ml}$, and 
Table I. Comparison of baseline characteristics between control and PCNSL groups.

\begin{tabular}{|c|c|c|c|c|}
\hline Clinicopathological characteristics & Control group & PCNSL group & $\chi^{2} / \mathrm{H}$ & P-value \\
\hline \multicolumn{5}{|l|}{$\operatorname{Sex}^{\mathrm{a}}$} \\
\hline Female & $20(37.7)$ & $21(55.3)$ & \multirow[t]{2}{*}{2.746} & \multirow[t]{2}{*}{0.097} \\
\hline Male & $33(62.3)$ & $17(44.7)$ & & \\
\hline \multicolumn{5}{|l|}{ Anatomical site ${ }^{\mathrm{a}}$} \\
\hline Cerebellum & $11(20.8)$ & $3(7.9)$ & \multirow[t]{3}{*}{4.504} & \multirow[t]{3}{*}{0.105} \\
\hline Mixture & $11(20.8)$ & $14(36.8)$ & & \\
\hline Superatentorial region & $31(58.5)$ & $21(55.3)$ & & \\
\hline \multicolumn{5}{|l|}{ Number of lesions $\mathrm{s}^{\mathrm{a}}$} \\
\hline 1 & $40(75.5)$ & $21(55.3)$ & \multirow[t]{3}{*}{4.912} & \multirow[t]{3}{*}{0.086} \\
\hline 2 & $4(7.5)$ & $8(21.1)$ & & \\
\hline$\geq 3$ & $9(17.0)$ & $9(23.7)$ & & \\
\hline \multicolumn{5}{|l|}{ Radiographic diagnosis $^{\mathrm{a}}$} \\
\hline Non-PCNSL & $50(94.3)$ & $21(55.3)$ & \multirow[t]{2}{*}{19.708} & \multirow[t]{2}{*}{$<0.001$} \\
\hline PCNSL & $3(5.7)$ & $17(44.7)$ & & \\
\hline \multicolumn{5}{|l|}{ Pandy test ${ }^{\mathrm{a}}$} \\
\hline- & $34(64.2)$ & $32(84.2)$ & \multirow[t]{3}{*}{-} & \multirow[t]{3}{*}{$0.071^{\mathrm{c}}$} \\
\hline+ & $18(34.0)$ & $6(15.8)$ & & \\
\hline++ & 1 (1.9) & $0 \quad(0.0)$ & & \\
\hline Age, years ${ }^{\mathrm{b}}$ & $52(43-57)$ & $55.5(52-63)$ & 10.491 & 0.001 \\
\hline $\mathrm{KPS}^{\mathrm{b}}$ & $80(80-90)$ & $70(60-90)$ & 14.621 & $<0.001$ \\
\hline Total tumor volume, $\mathrm{cm}^{3 \mathrm{~b}}$ & $3.444(1.918-8.456)$ & $4.948(2.985-12.353)$ & 1.129 & 0.288 \\
\hline Serum LDH, U// ${ }^{\mathrm{b}}$ & $225.6(165-508)$ & $208(161-550)$ & 0.077 & 0.782 \\
\hline Serum IL-6, pg/ml ${ }^{\mathrm{b}}$ & $2.2(1.50-5.26)$ & $2.16(1.50-5.26)$ & 0.000 & 0.983 \\
\hline Serum IL-10, pg/ml ${ }^{\mathrm{b}}$ & $1.115(1.00-2.04)$ & $2.18(1.00-3.52)$ & 5.130 & 0.024 \\
\hline CSF IL-6, pg/ml ${ }^{\mathrm{b}}$ & $6.87(5.14-14.78)$ & $6.18(4.14-25.60)$ & 0.006 & 0.936 \\
\hline CSF IL-10, pg/ml ${ }^{\mathrm{b}}$ & $2.61(2.01-3.08)$ & $32(21.90-156.00)$ & 60.463 & $<0.001$ \\
\hline CSF cell count ${ }^{\mathrm{b}}$ & $0.005(0.003-0.015)$ & $0.01(0.003-0.021)$ & 0.678 & 0.410 \\
\hline CSF Leukocyte count, $\times 10^{9} / 1^{b}$ & $0.002(0.001-0.005)$ & $0.0045(0.002-0.012)$ & 5.541 & 0.019 \\
\hline CSF LDH, U/1 & $18.5(10.9-23.5)$ & $14.9(10.9-24.2)$ & 0.233 & 0.629 \\
\hline $\mathrm{CSF}$ glucose, $\mathrm{mmol} / \mathrm{l}^{\mathrm{b}}$ & $3.46(3.18-3.93)$ & $4.32(3.93-4.95)$ & 23.217 & $<0.001$ \\
\hline CSF total protein, $\mathrm{g} / \mathrm{l}^{\mathrm{b}}$ & $0.34(0.20-0.57)$ & $0.38(0.32-0.57)$ & 3.460 & 0.063 \\
\hline Ratio of IL-10 to IL-6 in serum ${ }^{\mathrm{b}}$ & $0.667(0.246-0.815)$ & $0.667(0.407-1.393)$ & 1.754 & 0.185 \\
\hline Ratio of IL-10 to IL-6 in CSF & $0.341(0.214-0.526)$ & $7.880(5.573-11.672)$ & 56.867 & $<0.001$ \\
\hline
\end{tabular}

${ }^{a}$ Data presented as n (\%). ${ }^{b}$ Data presented as median and interquartile range (IQR). ${ }^{c}$ Data analyzed using Fisher's exact test. PCNSL, primary central nervous system lymphoma; KPS, Karnofsky performance score; LDH, lactate dehydrogenase; CSF, cerebrospinal fluid; IL, interleukin; $\mathrm{IQR}$, interquartile range.

the sensitivity, specificity, PPV and NPV of this cut-off value were $97.4,100,100$ and $98.1 \%$, respectively.

In the PCNSL group, 34 out of 35 cases (97.1\%) showed CSF IL-10 levels above the cut-off value. The CSF IL-10 level of 1 patient was below the cut-off value due to steroid treatment, with the CSF IL-6 and IL-10 levels being 3.55 and $1.88 \mathrm{ng} / \mathrm{ml}$, respectively. At 2 weeks post-withdrawal of steroid treatment, the CSF IL-6 and IL-10 levels increased to 4.23 and $57.8 \mathrm{pg} / \mathrm{ml}$, respectively, suggesting that steroid treatment can cause false-negative outcomes. Another case diagnosed as left occipitoparietal lymphadenoma showed a CSF IL-6 level of $69.69 \mathrm{pg} / \mathrm{ml}$ and a CSF IL-10 level of $1.56 \mathrm{pg} / \mathrm{ml}$, but the histological examination after craniotomy revealed a tuberculous granuloma. In general, the diagnostic efficacy of IL-6 levels was substantially lower than that of IL-10 levels.

Relevance of CSF IL-6 or IL-10 levels to the number of tumor lesions and imaging and histological manifestations. Among the 53 cases with a histological diagnosis of non-PCNSL, 50 were primarily detected by MRI, resulting in a specificity of $94.3 \%$. By contrast, among the 38 PCNSL cases, 17 were primarily detected by MRI with a sensitivity of $44.7 \%$. Among these, 3 were confirmed by MRI scans and IL-10 assessment without confirmation through biopsy, and the PET-CT assessments in these cases revealed hypermetabolic 
A

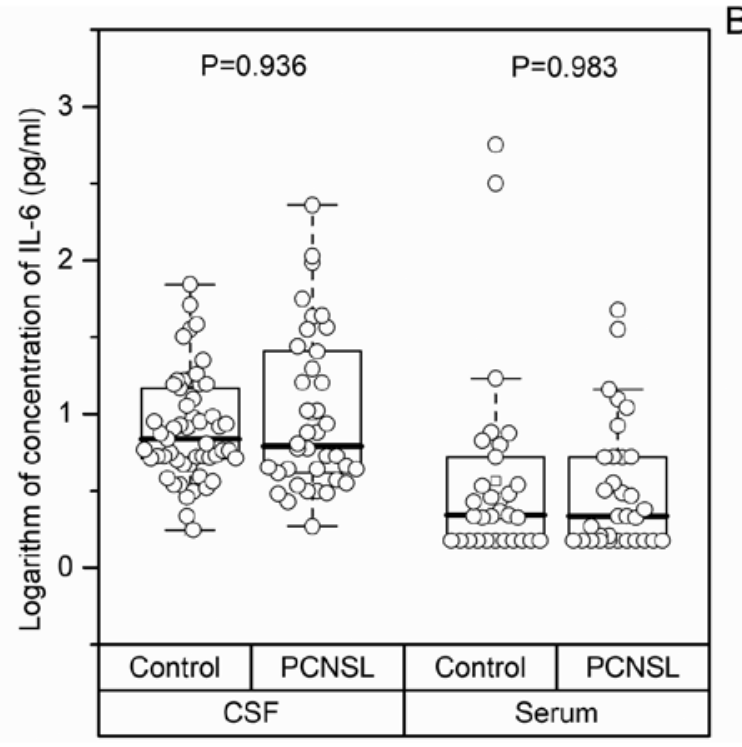

B

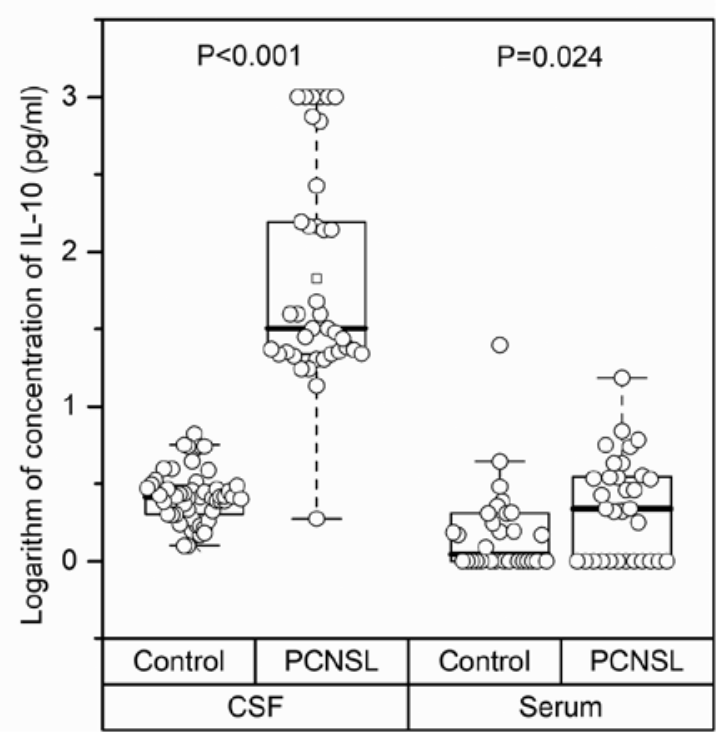

Figure 1. Distribution of (A) IL-6 and (B) IL-10 levels in the serum and CSF in the control and PCNSL groups. IL, interleukin; CSF, cerebrospinal fluid; PCNSL, primary central nervous system lymphoma.

foci. A total of 9 cases exhibited characteristics of DLBCL on histological examination. Among the 12 PCNSL cases with regular follow-up assessments, 4 (33.3\%) were primarily and $5(41.7 \%)$ were secondarily detected by MRI scan, while the remaining 3 cases were diagnosed as other tumors. As shown in Fig. 3, the low sensitivity of MRI for the diagnosis of PCNSL was substantially compensated by CSF IL-10 evaluations. In the 38 PCNSL cases, CSF IL-10 concentration rose significantly along with an increase in the number of tumor lesions [Number of tumor lesion: 1 vs. 2 vs. $\geq 3$, median (IQR): 24.15 (21.71-39.50) vs. 88.75 (21.81-206.50) vs. 697.00 (39.50-1,000.0), $\mathrm{P}=0.024]$. However, there was no significant correlation between CSF IL-6 $(\mathrm{P}=0.117)$, serum IL-6 $(\mathrm{P}=0.768)$, and serum IL-10 levels $(\mathrm{P}=0.554)$ and tumor lesion number (Fig. 4). Nevertheless, tumor size correlated positively with both serum and CSF IL-10 levels ( $\mathrm{r}=0.730$, $\mathrm{P}<0.001 ; \mathrm{r}=0.382, \mathrm{P}=0.018$; Fig. 5).

Role of CSF IL-10 levels in predicting the curative effect and prognosis of PCNSL. Among the 38 patients with PCNSL, 12 were followed up regularly, including 6 males and 6 females. The MRI scans primarily showed 4 cases of PCNSL. Tumors were located in the cerebellum, supratentorial region or at both sites in 2, 5 and 5 cases, respectively, while 1, 2 and 3 tumor lesions were present in 7, 3 and 2 cases, respectively. The follow-up periods ranged from 1 to 27 months, with a median period of 4 months, during which 4 cases showed disease progression and 3 patients died. The median PFS time was 8 months (95\% CI, 3.94-12.06), and the median OS time was 17.5 months (95\% CI, 11.55-23.45).

Case 2, which involved the most follow-up examinations, was diagnosed as left frontal glioblastoma by MRI before the operation. However, the CSF IL-10 level of the patient was $683.4 \mathrm{pg} / \mathrm{ml}$, indicating the presence of lymphadenoma. The case was then confirmed to be intracranial primary diffuse large B-cell lymphoma outside the germinal center by histological examination after craniotomy (T1; Fig. 6). The post-operative MRI indicated that the tumor had been CR, and this was accompanied by a reduction in the CSF IL-10 level to $20.2 \mathrm{pg} / \mathrm{ml}$ (T2; Fig. 6). The patient received two courses of chemotherapy with R $\left(375 \mathrm{mg} / \mathrm{m}^{2} \mathrm{~d} 1\right)$, H-MTX $\left(4 \mathrm{~g} / \mathrm{m}^{2}, \mathrm{~d} 1\right)$ and TMZ $\left(150 \mathrm{mg} / \mathrm{m}^{2}, \mathrm{~d} 1-5\right)$, and then underwent evaluation of CSF IL-10 levels, which had reached $745 \mathrm{pg} / \mathrm{ml}$. The immediate enhanced MRI scan showed a lesion with a size of $1.2 \times 0.9 \mathrm{~cm}$ beside the left lateral ventricle that appeared hypermetabolic on PET-CT, indicating tumor relapse (T3; Fig. 6). Therefore, the treatment was changed to whole-brain radiotherapy at $23.4 \mathrm{~Gy}$, with a local dose of up to $45 \mathrm{~Gy}$ in a total number of 13 fractions. The CSF IL-10 level reduced to $32 \mathrm{pg} / \mathrm{ml}$, corresponding to the CR observed on MRI (T4; Fig. 6). After 4 courses of chemotherapy with rituximab (R, $375 \mathrm{mg} / \mathrm{m}^{2} \mathrm{~d} 1$ ), high-dose methotrexate (H-MTX, $\left.4 \mathrm{~g} / \mathrm{m}^{2}, \mathrm{~d} 1\right)$ and TMZ $\left(150 \mathrm{mg} / \mathrm{m}^{2}, \mathrm{~d} 1-5\right)$, the CSF IL-10 level had reduced to $17.5 \mathrm{pg} / \mathrm{ml}$, with MRI showing CR (T5; Fig. 6). However, after another two courses with the same regimen, the patient experienced dizziness, which was associated with a corresponding increase in the CSF IL-10 level to $146 \mathrm{pg} / \mathrm{ml}$ and the presence of cloudy-like edema on the left frontal and basal ganglia zone on MRI (T6; Fig. 6). Therefore, a chemotherapy regimen based on rituximab and high-dose methotrexate was recommended, but the patient rejected this treatment Due to financial reasons and as no lesion was observed on MRI. At 1 month post-discharge from the hospital, the condition of the patient continued to deteriorate and symptoms of lethargy and a cough appeared; therefore, the patient was readmitted to the hospital. At this point, the CSF IL-10 level had increased to $>1,000 \mathrm{pg} / \mathrm{ml}$, and MRI showed large cloud-like edema with an irregular potentiated nodular focus in the left frontal and bilateral basilar ganglia zones (T7; Fig. 6). The patient exhibited extremely rapid disease progression and died with bedsores and a pulmonary fungal infection.

In PCNSL patients, the variations in CSF IL-10 levels were in line with the tumor size on MRI imaging. High levels of CSF IL-10 before therapy was associated closely with low 
Table II. AUCs and corresponding 95\% confidential intervals of serum IL-10 and four other CSF biochemical indicators for discriminating primary central nervous system lymphoma from control cases.

\begin{tabular}{|c|c|c|}
\hline Factors tested & AUC (95\% CI) & P-value \\
\hline Serum IL-10 ${ }^{\mathrm{a}}$ & $0.660(0.521-0.800)$ & 0.030 \\
\hline CSF IL-10 & $0.980(0.940-1.000)$ & 0.000 \\
\hline CSF Leukocyte count & $0.644(0.528-0.760)$ & 0.020 \\
\hline CSF glucose & $0.797(0.705-0.890)$ & 0.000 \\
\hline Ratio of CSF IL-10 to IL-6 & $0.965(0.924-1.000)$ & 0.000 \\
\hline
\end{tabular}

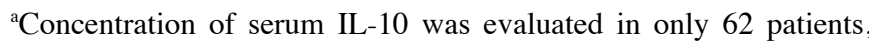
and all other biochemical indicators in CSF were determined in the whole population. CSF, cerebrospinal fluid; IL, interleukin; AUC, area under the curve.

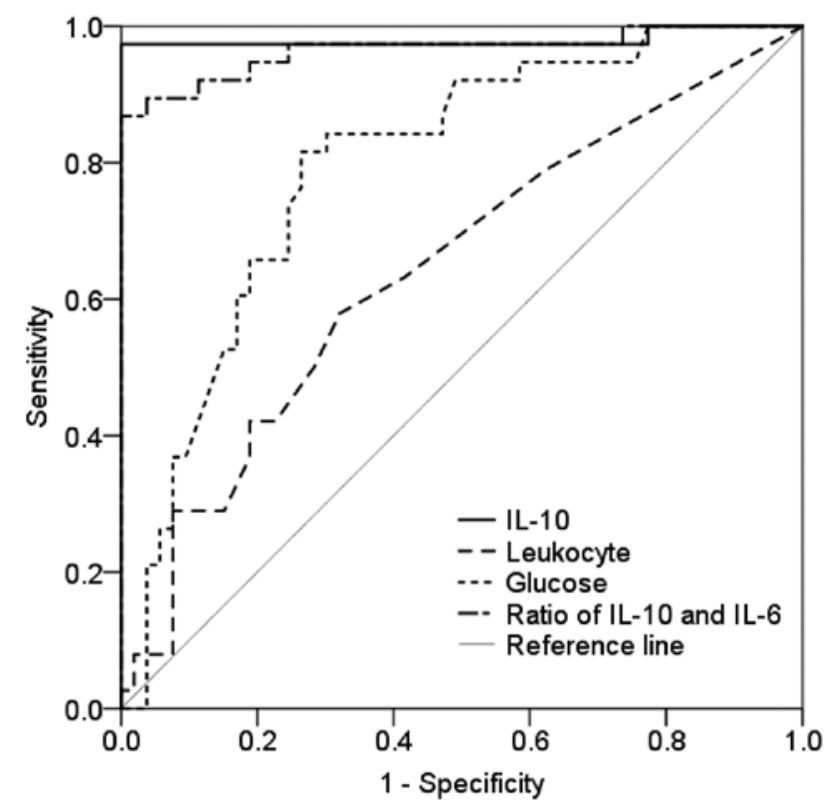

Figure 2. Receiver operating characteristic curve analysis of the contributions of serum and CSF IL-10 levels, CSF leukocyte count, CSF glucose concentration and the ratio of IL-10 to IL-6 levels in the CSF to the diagnosis of PCNSL. IL, interleukin; CSF, cerebrospinal fluid.

KPS scores and short PFS or OS times. In cases 1, 2, 3, 6 and 12, the increase in CSF IL-10 levels appeared earlier than MRI features after treatment, indicating that this biomarker held substantial value in predicting tumor relapse. CSF IL-10 levels $>138 \mathrm{pg} / \mathrm{ml}$ before therapy occurred with markedly low KPS scores. A total of 6 cases with CSF IL-10 levels $>1,000 \mathrm{pg} / \mathrm{ml}$ showed rapid disease progression, signifying that CSF IL-10 levels $>1,000 \mathrm{pg} / \mathrm{ml}$ could be a crucial sign of a poor prognosis.

\section{Discussion}

Il-6 (14) and IL-10 $(18,19)$ have been shown to be associated with the occurrence and prognosis of lymphoid malignancies. In previous studies, high serum IL-6 and IL-10 levels were considered to be independent predictors of the prognosis of invasive and systemic NHL $(30,31)$. The cutoff value for serum IL-10 in 91 patients in the present study was $2.07 \mathrm{pg} / \mathrm{ml}$, while the sensitivity, specificity, and positive and negative predictive values of serum IL-10 levels for the diagnosis of PCNSL were 59.4, 83.3, 79.2 and 65.8, respectively. The serum IL-6 level was lower than the serum IL-10 level $(\mathrm{P}=0.030)$, and the serum IL-10 level was lower than the CSF IL-10 level $(\mathrm{P}<0.001)$. In the present cohort, 1 patient presented with left occipital lymphoma by MRI examination. The levels of CSF IL-6 and IL-10 in this patient were 69.69 and $1.56 \mathrm{pg} / \mathrm{ml}$, respectively, and histological examination showed tuberculous granuloma. Sasayama et al (10) found that when the cutoff value was $4.0 \mathrm{pg} / \mathrm{ml}$, the sensitivity and specificity of the CSF IL-6 level for diagnosing PCNSL were 77 and 63\%, respectively. Serum and CSF IL-6 levels have certain reference value in the diagnosis of PCNSL, but have low specificity (32).

The CSF IL-10 level has a high sensitivity and specificity in the diagnosis of PCNSL $(10,11)$. Among the 91 patients recruited in the present study, 38 had confirmed PCNSL and 53 had other brain tumors. In the 38 patients of the PCNSL group, the CSF IL-10 concentration increased significantly with increasing number of lesions (1 vs. 2 vs. $\geq 3,24.15$ (21.71-39.50) vs. 88.75 (21.81-206.50) vs. 697.00 (39.50-1,000.0); $\mathrm{P}=0.024)$. There were significant positive correlations between total tumor volume and serum or CSF IL-10 ( $\mathrm{r}=0.730, \mathrm{P}<0.001$; $\mathrm{r}=0.382, \mathrm{P}=0.018$ ). Considering the anti-inflammatory properties of IL-10, a continuously high level of CSF IL-10 may trigger relatively strong immune activation, promote cell proliferation and inhibit cell apoptosis $(33,34)$. It can be inferred that CSF IL-10 is produced by tumor cells, and that the higher the level of CSF IL-10, the greater the tumor burden, the lower the KPS score and the worse the prognosis.

According to the Youden index, the cutoff value for serum IL-10 in the 91 patients of the present study was $2.07 \mathrm{pg} / \mathrm{ml}$. This cutoff value was sensitive (59.4\%) and specific (83.3\%), with a PPV of $79.2 \%$ and a NPV of $65.8 \%$. The cutoff value for CSF IL-10 was $10.13 \mathrm{pg} / \mathrm{ml}$, and this cutoff value was sensitive $(97.4 \%)$ and specific (100\%), with a PPV of $100 \%$ and an NPV of $98.1 \%$. For the differential diagnosis of PCNSL, CSF IL-10 has been recognized to be significantly more effective than serum IL-10 in terms of specificity and sensitivity, as the former can prevent a false-positive diagnosis from interfering with the clinical application of various biomarkers of PCNSL, including IL-10, which has been previously reported as occurring (10,11,35-41). Whitcup et al (36) first found that the ratio of IL-10 to IL-6 in the CSF associated with CNS lymphoma was increased, and Salmaggi et al (37) reported that the CSF IL-10 concentration in PCNSL was higher than that in other CNS tumors. In the present study, only solid tumor patients suspected to have PCNSL were included, and the specificity of CSF IL-10 in the diagnosis of PCNSL was $100 \%$, which is consistent with previous reports $(9,10)$. The most recent review article also supports the high specificity of CSF IL-10 in the diagnosis of PCNSL.

The influence of hormones on CSF IL-10 remains to be elucidated. Among the 53 patients with brain tumors other than PCNSL in the present study, 14 showed CSF IL-10 levels lower than the minimal limit of the test $(2 \mathrm{pg} / \mathrm{ml})$. Conversely, only 1 PCNSL patient had similarly low levels of CSF IL-10. 


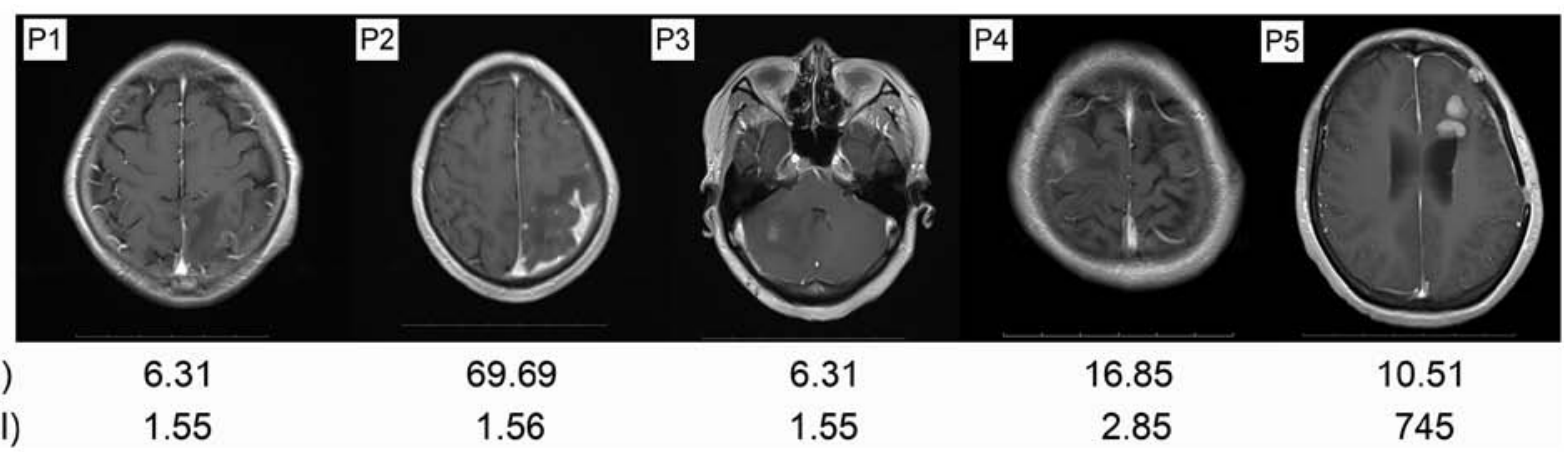
IL-6 (pg/ml)
6.31
1.56
6.31
2.85
745

Figure 3. Evaluation of CSF IL-10 levels enhance the positive detection of PCNSL by MRI. P1, left parietal metastatic adenocarcinoma; P2, left parietal tuberculous granuloma; P3, cerebellar diffuse astrocytoma (WHO grade II); P4, right parietal low differentiated adenocarcinoma; P5, left frontal PCNSL. The values below the MRI images are the CSF IL-6 and IL-10 levels of the corresponding patients. IL, interleukin; CSF, cerebrospinal fluid; PCNSL, primary central nervous system lymphoma.

A

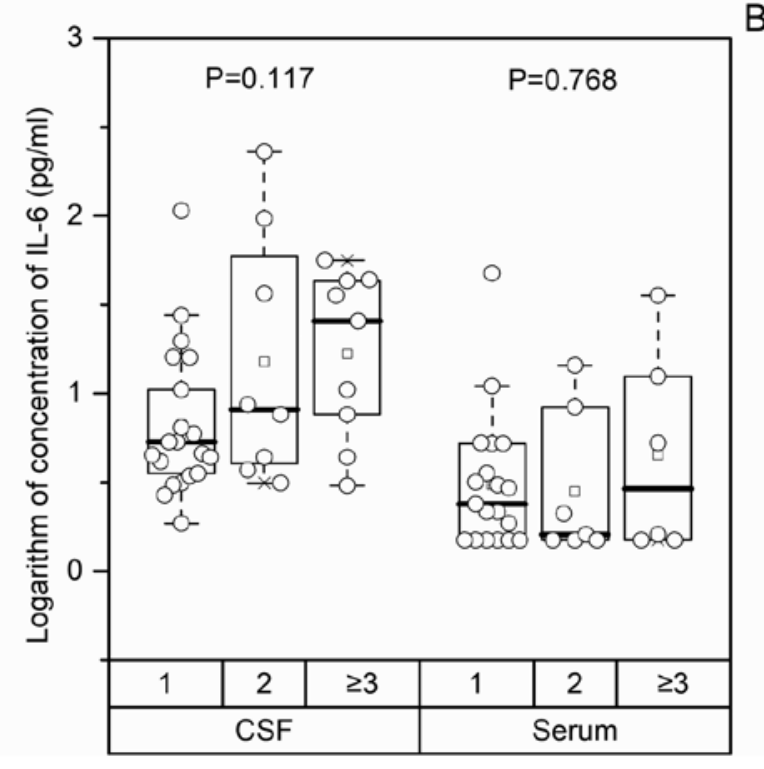

B

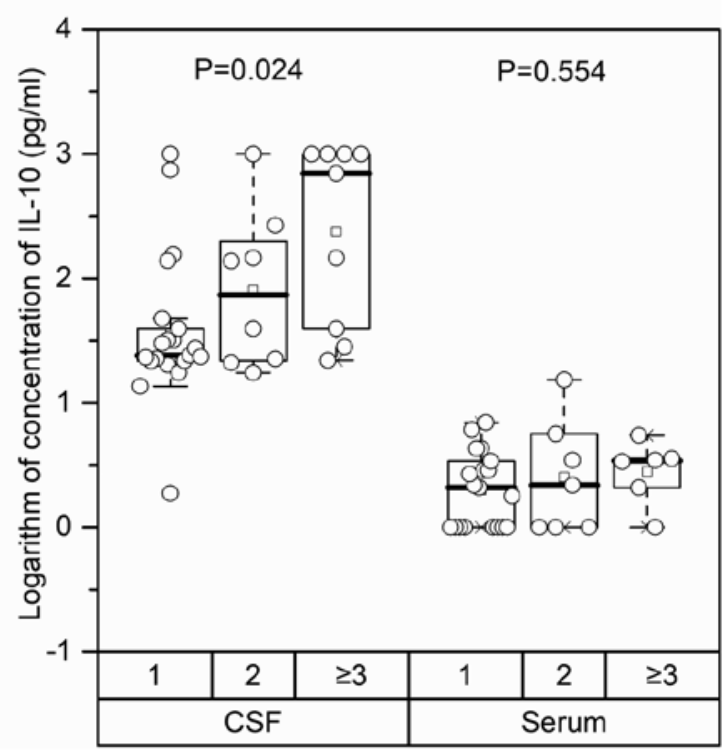

Figure 4. Association between serum or CSF (A) IL-6 or (B) IL-10 levels and the number of tumor lesions. IL, interleukin; CSF, cerebrospinal fluid.

The MRI scan of this patient was indicative of PCNSL, and PET-CT examination highlighted the hypermetabolic lesion. This patient was undergoing high-dose steroid treatment for management of a constantly deteriorating condition, and the CSF IL-6 and IL-10 levels after initiation of this treatment were 3.55 and $1.88 \mathrm{pg} / \mathrm{ml}$ respectively. However, 3 weeks after withdrawal of steroid therapy, the CSF IL-6 level increased to $4.23 \mathrm{pg} / \mathrm{ml}$, while the CSF IL-10 level rose to $57.8 \mathrm{pg} / \mathrm{ml}$. Steroids can suppress the survival of malignant cells, but the tumor cells may recover soon after steroid treatment is halted. Since patients receiving steroid treatment may show reduced CSF IL-6 and IL-10 levels (36), it is necessary to assess these levels again 2 to 3 weeks after withdrawing steroid therapy. In addition, due to the fragility of malignant lymphoma cells, these cells are not easily detected in cytopathological examinations. The present study involved only one case with detectable lymphoma cells in the CSF smear.

Although an optimal treatment strategy for PCNSL based on high-dose methotrexate has not yet been determined, whole-body radiotherapy and stem cell transplantation are currently the most important treatment methods $(7,42)$. In the present study, 35 PCNSL patients were recruited from different centers and received different treatments, making it difficult to summarize PFS and OS data. However, 12 patients, including 2 who received the most follow-up examinations, were regularly followed up, with a 14-month interval from diagnosis to death. These results indicate that the CSF IL-10 level might be indicative of the tumor burden, and that the higher the IL-10 level, the more invasive the tumor, the stronger the cellular immune response and the shorter the survival time. In addition, the increased level of CSF IL-10 is closely associated with poor survival and is an independent risk factor for predicting the prognosis of PCNSL $(20,36,43)$. More importantly, in the present study, the increase in CSF IL-10 levels appeared earlier than the MRI abnormalities, suggesting the CSF IL-10 may have value in monitoring the treatment of tumor recurrence or clinical decision-making. In addition, increased CSF IL-10 levels with increased tumor burden hinted that this cytokine might be produced by tumor cells (44). However, high secretion of IL-10 by cells was difficult to detect in the present study, as CSF IL-10 levels $>1,000 \mathrm{pg} / \mathrm{ml}$ were associated with the rapidly deteriorating 
A

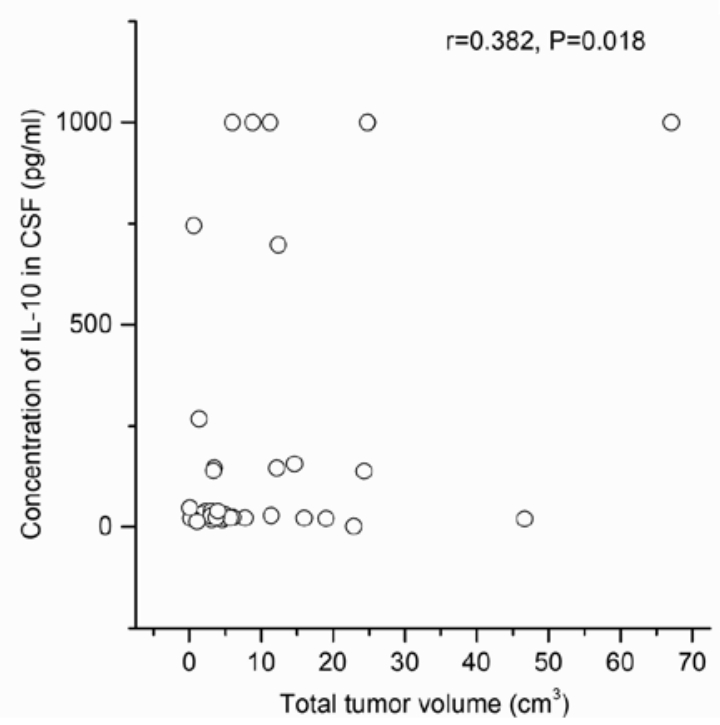

B

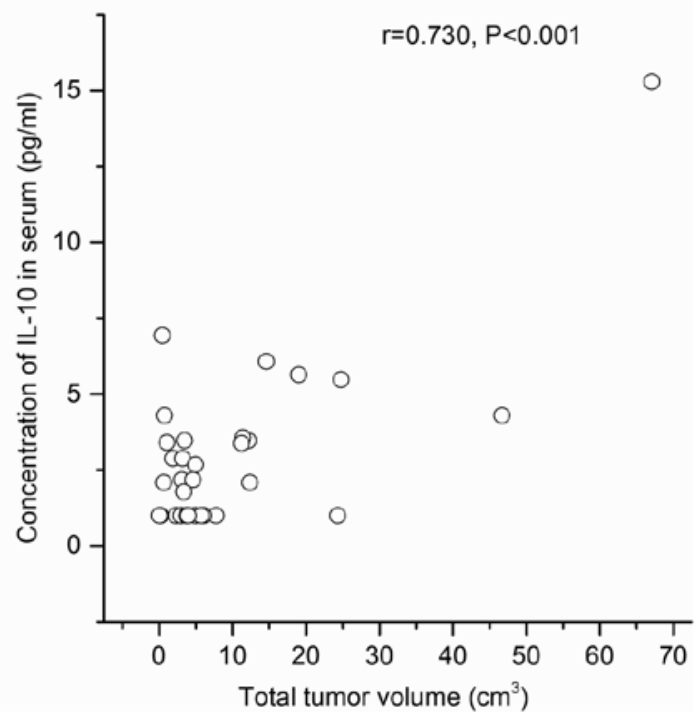

Figure 5. Correlation of tumor volume with serum or CSF IL-6 (A) or IL-10 levels (B) ( $r=0.730, P<0.001 ; r=0.382, P=0.018)$. IL, interleukin; CSF, cerebrospinal fluid.
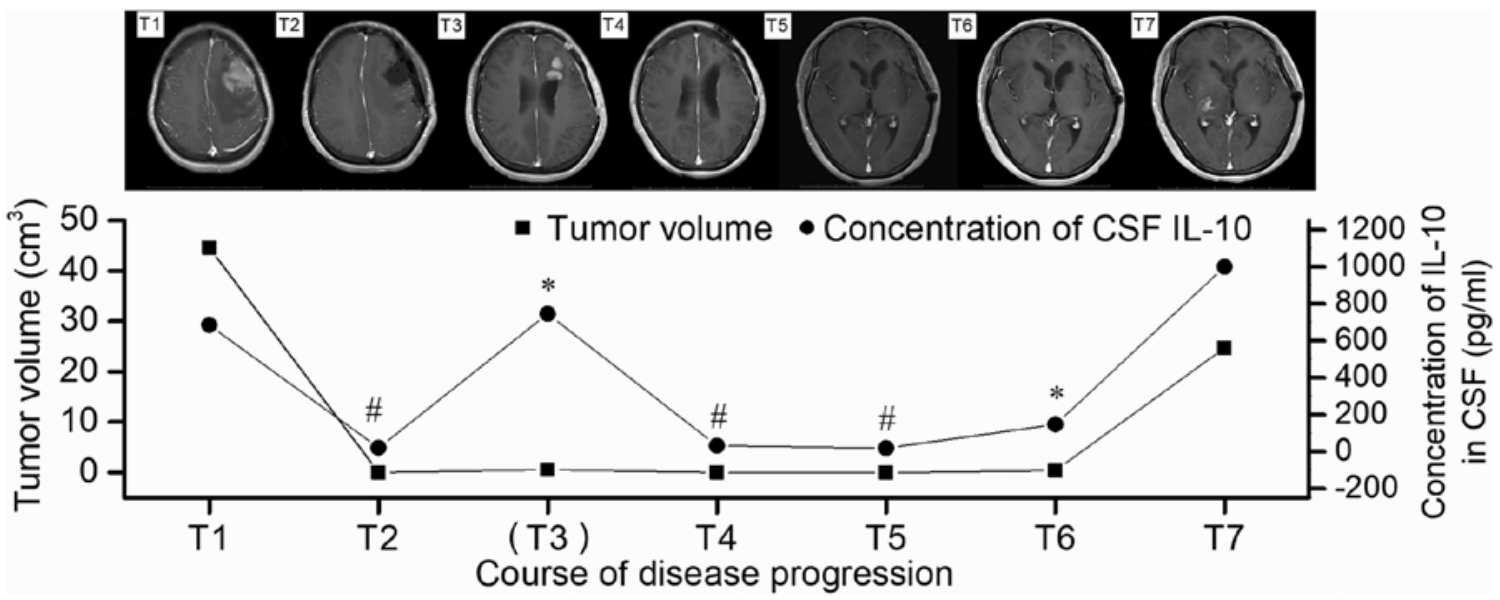

Figure 6. Illustration of changes in MRI of intracranial lesions and dynamics of CSF IL-10 levels in one patient during disease progression. The upper panel shows a series of MRI images depicting tumor regression and relapse at different time points. The lower panel demonstrates the numeric tracks of tumor volume and CSF IL-10 levels at the corresponding time points. Preoperative MRI indicated glioblastoma, but the IL-10 and immunohistochemistry test confirmed the diagnosis as PCNSL (T1). The symbol \# indicates time points at which the intracranial tumor reached complete remission, as well as almost undetectable levels of IL-10 in the CSF (T2, T4 and T5). The asterisk symbols indicate that the concentration of IL-10 in CSF was markedly elevated when the tumor recurred, but that the tumor volumes determined by MRI only slightly increased (T3, T6 and T7). The square points represent tumor volume and the circles represent the concentration of CSF IL-10. IL, interleukin; CSF, cerebrospinal fluid.

condition of the patient, which means that the levels of CSF IL-10, in addition to having an application in monitoring the tumor state, may also be useful in predicting prognosis (22). Biomarkers of these characteristics make CSF IL-10 superior to other conventional tumor biomarkers, as these conventional markers are essentially tumor cell metabolites and therefore can be modified only on behalf of the tumor itself. However, a previous study reported that IL-10 can inhibit tumor angiogenesis, promote cytotoxic factor generation, induce tumor elimination and improve survival rate (20).

IL-10 levels $\geq 138$ and $\geq 1,000 \mathrm{pg} / \mathrm{ml}$ before and after treatment, respectively, were important predictors of a poor prognosis in the present study, which also indicates that CSF IL-10 expressed at high levels in the CSF accelerated the growth of tumor cells resistant to both radiotherapy and chemotherapy. First, IL-10 may be produced by malignant B lymphocytes, which strongly stimulates the proliferation and differentiation of B cells, and promotes the occurrence and development of lymphoma (30). On the other hand, a high level of IL-10 is associated with immunosuppressive effects, which can suppress the antitumor immune response and interfere with immune monitoring (21). An experimental study also found that IL-10 could protect cells, promote cell growth via autocrine or paracrine mechanisms to activate NHL, promote proliferation, inhibit apoptosis and promote tumor cell growth (45). A drug susceptibility test showed that IL-10 is crucial in NHL resistance (33), which suggests that IL-10 not only is very important during tumorigenesis, 
but also plays a promotive role in the growth of tumor, as IL-10- or IL-10 receptor-specific treatment may be an effective treatment strategy for PCNSL (46). An IL-10 concentration $>1,000 \mathrm{pg} / \mathrm{ml}$ is considered a high-risk factor for PCNSL, and targeting IL-10 will improve treatment intensity and patient PFS and OS times, and reduce the CNS progression rate (47), which makes IL-10 worthy of further in-depth study.

In regard to the limitations of the present study, the small sample size was a primary limitation. Since PCNSL is a rare disease of the brain, a single center will normally encounter only a few cases, which may lead to data bias. The unitary disease category was another limitation of the study. Due to its prospective nature, this study recruited only patients with suspected PCNSL shown by MRI. However, some reports have shown that increased levels of CSF IL-10 are also found in idiopathic inflammatory disease to a certain extent (41). Therefore, before drawing further conclusions, studies with broader disease categories and larger sample sizes should be conducted. The third limitation of the present work was the incomplete basic clinical information of the patients, which could be addressed by closer follow-up.

In summary, even with the numerous shortcomings, the present study proved that the CSF IL-10 level was the most significant biomarker for patients with suspected PCNSL by neuroimaging and the presence of a tumor mass. Additionally, the CSF IL-10 level served as an independent predictor for prognosis that was closely associated with the poor survival of PCNSL patients. These findings provide a reference for clinicians to identify more effective therapeutic strategies. Further prospective, multicenter studies would be helpful for clarifying these issues in the future.

\section{Acknowledgements}

Not applicable.

\section{Funding}

No funding was received.

\section{Availability of data and materials}

The datasets used and/or analyzed during the current study are available from the corresponding author on reasonable request.

\section{Authors' contributions}

MG, CC, and GW conceived and designed the experiments. MG and YS performed the main experiments and analyzed the data. HX, ZW and XD participated in the measurement and analysis of IL-6 and IL-10 in the blood and CSF. MG and YS wrote the manuscript. All authors read and approved the final manuscript.

\section{Ethics approval and consent to participate}

This study was approved by The Medical Ethics Committee at the Daping Hospital, Army Medical University (Third Military Medical University).

\section{Patient consent for publication}

Written informed consent for the publication of images and all other clinical data is obtained from the enrolled patients.

\section{Competing interests}

The authors declare that they have no competing interests.

\section{References}

1. Su M, Huang D, Sun L, Dong Z, Wu L and Yu S: A diagnostic challenge of primary Central nervous system lymphoma: From the eyes to the brain. Int J Neurosci: 1-7, 2020 (Epub ahead of print). doi: 10.1080/00207454.2020.1773822.

2. Bataille B, Delwail V, Menet E, Vandermarcq P, Ingrand P, Wager M, Guy G and Lapierre F: Primary intracerebral malignant lymphoma: Report of 248 cases. J Neurosurg 92: 261-266, 2000.

3. Hartmann M, Heiland S, Harting I, Tronnier VM, Sommer C, Ludwig R and Sartor K: Distinguishing of primary cerebral lymphoma from high-grade glioma with perfusion-weighted magnetic resonance imaging. Neurosci Lett 338: 119-122, 2003.

4. Bertaux M, Houillier C, Edeline V, Habert MO, Mokhtari K, Giron A, Bergeret S, Hoang-Xuan K, Cassoux N, Touitou V, et al: Use of FDG-PET/CT for systemic assessment of suspected primary central nervous system lymphoma: A LOC study. J Neurooncol 148: 343-352, 2020.

5. Josephson SA, Papanastassiou AM, Berger MS, Barbaro NM, McDermott MW, Hilton JF, Miller BL and Geschwind MD: The diagnostic utility of brain biopsy procedures in patients with rapidly deteriorating neurological conditions or dementia. J Neurosurg 106: 72-75, 2007.

6. Khatab S, Spliet W and Woerdeman PA: Frameless image-guided stereotactic brain biopsies: Emphasis on diagnostic yield. Acta Neurochir (Wien) 156: 1441-1450, 2014.

7. Ferreri AJM, Cwynarski K, Pulczynski E, Fox CP, Schorb E, La Rosee P, Binder M, Fabbri A, Torri V, Minacapelli E, et al: Whole-brain radiotherapy or autologous stem-cell transplantation as consolidation strategies after high-dose methotrexate-based chemoimmunotherapy in patients with primary CNS lymphoma: Results of the second randomisation of the International Extranodal Lymphoma Study Group-32 phase 2 trial. Lancet Haematol 4: e510-e523, 2017.

8. Gilbert MR, Wang M, Aldape KD, Stupp R, Hegi ME, Jaeckle KA, Armstrong TS, Wefel JS, Won M, Blumenthal DT, et al: Dose-dense temozolomide for newly diagnosed glioblastoma: A randomized phase III clinical trial. J Clin Oncol 31: 4085-4091, 2013.

9. Viaccoz A, Ducray F, Tholance Y, Barcelos GK, Thomas-Maisonneuve L, Ghesquieres H, Meyronet D, Quadrio I Cartalat-Carel S, Louis-Tisserand G, et al: CSF neopterin level as a diagnostic marker in primary central nervous system lymphoma. Neuro Oncol 17: 1497-1503, 2015.

10. Sasayama T, Nakamizo S, Nishihara M, Kawamura A, Tanaka H, Mizukawa K, Miyake S, Taniguchi M, Hosoda K and Kohmura E: Cerebrospinal fluid interleukin-10 is a potentially useful biomarker in immunocompetent primary central nervous system lymphoma (PCNSL). Neuro Oncol 14: 368-380, 2012.

11. Sasagawa $Y$, Akai T, Tachibana $\mathrm{O}$ and Iizuka $\mathrm{H}$ : Diagnostic value of interleukin-10 in cerebrospinal fluid for diffuse large B-cell lymphoma of the central nervous system. J Neurooncol 121: 177-183, 2015.

12. Baraniskin A, Kuhnhenn J, Schlegel U, Maghnouj A, Zollner H, Schmiegel W, Hahn S and Schroers R: Identification of microRNAs in the cerebrospinal fluid as biomarker for the diagnosis of glioma. Neuro Oncol 14: 29-33, 2012.

13. Geng M, Xiao H, Liu J, Song Y, Fu P, Cheng X, Zhang J and Wang G: The diagnostic role and dynamic changes in cerebrospinal fluid neopterin during treatment of patients with primary central nervous system lymphoma. Cancer Med 7: 3889-3898, 2018.

14. Hoekzema R, Murray PI, van Haren MA, Helle M and Kijlstra A: Analysis of interleukin-6 in endotoxin-induced uveitis. Invest Ophthalmol Vis Sci 32: 88-95, 1991

15. Salles G and Coiffier B: Inherited cytokine response and risk of lymphoma. Lancet Oncol 7: 3-4, 2006. 
16. Kurzrock R: The role of cytokines in cancer-related fatigue Cancer 92 (Suppl 6): S1684-S1688, 2001.

17. Rousset F, Garcia E, Defrance T, Peronne C, Vezzio N, Hsu DH, Kastelein R, Moore KW and Banchereau J: Interleukin 10 is a potent growth and differentiation factor for activated human B lymphocytes. Proc Natl Acad Sci USA 89: 1890-1893, 1992.

18. Fluckiger AC, Durand I and Banchereau J: Interleukin 10 induces apoptotic cell death of B-chronic lymphocytic leukemia cells. J Exp Med 179: 91-99, 1994.

19. Stewart JP, Behm FG, Arrand JR and Rooney CM: Differential expression of viral and human interleukin-10 (IL-10) by primary B cell tumors and B cell lines. Virology 200: 724-732, 1994.

20. Mocellin S, Marincola FM and Young HA: Interleukin-10 and the immune response against cancer: A counterpoint. J Leukoc Biol 78: 1043-1051, 2005.

21. Moore KW, de Waal MR, Coffman RL and O'Garra A: Interleukin-10 and the interleukin-10 receptor. Annu Rev Immunol 19: 683-765, 2001.

22. Mosser DM and Zhang X: Interleukin-10: New perspectives on an old cytokine. Immunol Rev 226: 205-218, 2008.

23. O'Garra A, Barrat FJ, Castro AG, Vicari A and Hawrylowicz C: Strategies for use of IL-10 or its antagonists in human disease. Immunol Rev 223: 114-131, 2008.

24. Frappaz D, Bonneville-Levard A, Ricard D, Carrie S, Schiffler C, Xuan KH and Weller M: Assessment of Karnofsky (KPS) and WHO (WHO-PS) performance scores in brain tumour patients: The role of clinician bias. Support Care Cancer: Aug 13, 2020 (Epub ahead of print). doi: 10.1007/s00520-020-05663-y.

25. Teusch W: Relationships between Pandy's test and syphilis reactions in the spinal fluid. Med Monatsschr 4: 290-291, 1950 (In Undetermined Language).

26. Louis DN, Perry A, Reifenberger G, von Deimling A, Figarella-Branger D, Cavenee WK, Ohgaki H, Wiestler OD, Kleihues P and Ellison DW: The 2016 World health organization classification of tumors of the central nervous system: A summary. Acta Neuropathol 131: 803-820, 2016.

27. Nabors LB, Portnow J, Ammirati M, Baehring J, Brem H, Butowski N, Fenstermaker RA, Forsyth P, Hattangadi-Gluth J, Holdhoff M, et al: NCCN guidelines insights: Central nervous system cancers, version 1.2017. J Natl Compr Canc Netw 15: 1331-1345, 2017

28. Sugita Y, Muta H, Ohshima K, Morioka M, Tsukamoto Y, Takahashi $\mathrm{H}$ and Kakita A: Primary central nervous system lymphomas and related diseases: Pathological characteristics and discussion of the differential diagnosis. Neuropathology 36 : 313-324, 2016

29. Fluss R, Faraggi D and Reiser B: Estimation of the Youden index and its associated cutoff point. Biometrical J 47: 458-472, 2005.

30. El Far M, Fouda M, Yahya R and El Baz H: Serum IL-10 and IL-6 levels at diagnosis as independent predictors of outcome in non-Hodgkin's lymphoma. J Physiol Biochem 60: 253-258, 2004

31. Duletić AN, Stifter S, Dvornik S, Skunca Z and Jonjić N: Correlation of serum IL-6, IL-8 and IL-10 levels with clinicopathological features and prognosis in patients with diffuse large B-cell lymphoma. Int J Lab Hematol 30: 230-239, 2008

32. Song Y, Zhang W, Zhang L, Wu W, Zhang Y, Han X, Yang C, Zhang L and Zhou D: Cerebrospinal Fluid IL-10 and IL-10/IL-6 as accurate diagnostic biomarkers for primary central nervous system large B-cell lymphoma. Sci Rep 6: 38671, 2016.

33. Alas S, Emmanouilides $C$ and Bonavida B: Inhibition of interleukin 10 by rituximab results in down-regulation of bcl-2 and sensitization of B-cell non-Hodgkin's lymphoma to apoptosis. Clin Cancer Res 7: 709-723, 2001.

34. Vega MI,Huerta-YepazS, Garban H,Jazirehi A,EmmanouilidesC and Bonavida B: Rituximab inhibits p38 MAPK activity in 2F7 BNHL and decreases IL-10 transcription: Pivotal role of p38 MAPK in drug resistance. Oncogene 23: 3530-3540, 2004.
35. van Westrhenen A, Smidt LCA, Seute T, Nierkens S, Stork ACJ, Minnema MC and Snijders TJ: Diagnostic markers for CNS lymphoma in blood and cerebrospinal fluid: A systematic review. Brit J Haematol 182: 384-403, 2018

36. Whitcup SM, Stark-Vancs V, Wittes RE, Solomon D, Podgor MJ, Nussenblatt RB and Chan CC: Association of interleukin 10 in the vitreous and cerebrospinal fluid and primary central nervous system lymphoma. Arch Ophthalmol 115: 1157-1160, 1997.

37. Salmaggi A, Eoli M, Corsini E, Gelati M, Frigerio S, Silvani A and Boiardi A: Cerebrospinal fluid interleukin-10 levels in primary central nervous system lymphoma: A possible marker of response to treatment? Ann Neurol 47: 137-138, 2000.

38. Rubenstein JL, Wong VS, Kadoch C, Gao HX, Barajas R, Chen L, Josephson SA, Scott B, Douglas V, Maiti M, et al: CXCL13 plus interleukin 10 is highly specific for the diagnosis of CNS lymphoma. Blood 121: 4740-4748, 2013.

39. Mabray MC, Barajas RF, Villanueva-Meyer JE, Zhang CA, Valles FE, Rubenstein JL and Cha S: The combined performance of ADC, CSF CXC chemokine ligand 13, and CSF interleukin 10 in the diagnosis of central nervous system lymphoma. AJNR Am J Neuroradiol 37: 74-79, 2016.

40. Nguyen-Them L, Costopoulos M, Tanguy ML, Houillier C, Choquet S, Benanni H, Elias-Shamieh R, Armand M, Faivre G, Glaisner S, et al: The CSF IL-10 concentration is an effective diagnostic marker in immunocompetent primary CNS lymphoma and a potential prognostic biomarker in treatment-responsive patients. Eur J Cancer 61: 69-76, 2016.

41. Ikeguchi R, Shimizu Y, Shimizu S and Kitagawa K: CSF and clinical data are useful in differentiating CNS inflammatory demyelinating disease from CNS lymphoma. Mult Scler 24: 1212-1223, 2018

42. Thiel E, Korfel A, Martus P, Kanz L, Griesinger F, Rauch M, Roth A, Hertenstein B, von Toll T, Hundsberger T, et al: High-dose methotrexate with or without whole brain radiotherapy for primary CNS lymphoma (G-PCNSL-SG-1): A phase 3, randomised, non-inferiority trial. Lancet Oncol 11: 1036-1047, 2010.

43. Sasayama T, Tanaka K, Mizowaki T, Nagashima H, Nakamizo S, Tanaka H, Nishihara M, Mizukawa K, Hirose T, Itoh T and Kohmura E: Tumor-associated macrophages associate with cerebrospinal fluid interleukin-10 and survival in primary central nervous system lymphoma (PCNSL). Brain Pathol 26: 479-487, 2016.

44. Dukers DF, Jaspars LH, Vos W, Oudejans JJ, Hayes D, Cillessen S, Middeldorp JM and Meijer CJ: Quantitative immunohistochemical analysis of cytokine profiles in Epstein-Barr virus-positive and -negative cases of Hodgkin's disease. J Pathol 190: 143-149, 2000.

45. Voorzanger N, Touitou R, Garcia E, Delecluse HJ, Rousset F, Joab I, Favrot MC and Blay JY: Interleukin (IL)-10 and IL-6 are produced in vivo by non-Hodgkin's lymphoma cells and act as cooperative growth factors. Cancer Res 56: 5499-5505, 1996.

46. Wu X, Hsu DK, Wang KH, Huang Y, Mendoza L, Zhou Y and Hwang ST: IL-10 is overexpressed in human cutaneous T-cell lymphoma and is required for maximal tumor growth in a mouse model. Leuk Lymphoma 60: 1244-1252, 2019.

47. Leppa S, Jorgensen J, Tierens A, Meriranta L, Ostlie I, de Nully BP, Fagerli UM,Larsen TS, Mannisto S, Munksgaard L, et al: Patients with high-risk DLBCL benefit from dose-dense immunochemotherapy combined with early systemic CNS prophylaxis. Blood Adv 4: 1906-1915, 2020.

This work is licensed under a Creative Commons Attribution-NonCommercial-NoDerivatives 4.0 International (CC BY-NC-ND 4.0) License. 